\title{
LINESTRENOL ADMINISTRADO EN FORMA CONTINUA COMO ANTICONCEPTIVO*
}

\author{
Dr. Jaime Botero Uribe** \\ Dr. José Aníbal Castañeda Leal** \\ Dr. Jaime Uribe Duque** \\ Sta. Clara Inés Arango Rico** \\ Sta. Margarita Gutiérrez Arboleda**
}

\section{Introducción}

La administración continua de compuestos progestacionales en microdosis ha sido estudiada por varios autores $(1,2)$ con resultados en general satisfactorios. Los escasos efectos colaterales, la poca variabilidad en los ciclos menstruales y la eficacia anticonceptiva, han hecho de esta modalidad de anticoncepción oral, una de las más promisorias $(2,3)$.

El linestrenol es una sustancia progestacional desarrollada por los Laboratorios Organón de Holanda y es 17 alfa etinil 4 estreno 1701 . Por más de 8 años se ha usado a la dosis de $2.5 \mathrm{mg}$. en combinación con 75 gamas de mestranol en tabletas para administrar durante 22 días de cada ciclo***, sin que haya mostrado ningún efecto tóxico.

Algunos estudios preliminares con linestrenol a la dosis de $0.5 \mathrm{mg} .{ }^{* * *}$ * han mostrado su utilidad como anticonceptivo. Salazar (4) administró el compuesto a 135 mujeres, por un total de 386 ciclos. En 43 de ellas, que tomaron la droga en forma ininterrumpida durante 6 ciclos o más, no ocurrieron embarazos. En 44 que las ingirieron entre 1 y 5 ciclos, ocurrieron dos embarazos. Se presentaron polimenorreas en un $30.83 \%$ de los casos e hipermenorreas en un $19.4 \%$. En un $5 \%$ la menstruación se acompañó de coágulos, en $1.8 \%$ ocurrió hipermenorrea; cólicos menstruales ocurrieron en un $15 \%$ y cefálea premenstrual en un $11.8 \%$.

Ravn (5) en un total de 3.553 ciclos estudiados en 361 mujeres, no tuvo ninguna falla atribuíble a la misma droga administrada en iguales dosis. De los efectos colaterales hemorragia por rompimiento o pintas fueron los más comunes, y se redujeron a un $2.6 \%$ entre los ciclos 4 y 12.

El mecanismo de acción de esta forma de administración del linestrenol parece estar muy íntimamente relacionada con cambios en el moco cervical que lo hacen hostil al paso

\footnotetext{
* Investigación patrocinada por la casa Organón de Oss, Holanda.

** Del Departamento de Obstetricia y Ginecología, Facultad de Medicina, Universidad de Antioquia.

$\star * *$ Lindiol, marca registrada.

$\star \star * *$ Exlutón.
} 
de los espermatozoides (6); también se ha encontrado que produce alteraciones en el estroma endometrial (7) $y$ que inhibe, aunque en forma inconsistente, la ovulación (8).

\section{Material y Métodos}

El trabajo se realizó mediante el ingreso en forma consecutiva de 308 mujeres que Ilenaron las siguientes condiciones:

a) Edad fértil y fertilidad demostrada.

b) Posibilidad de quedar embarazada.

c) Posibilidad de ser controlada con intervalos de un mes.

Se hizo una encuesta inicial a todas las mujeres que fueran candidatas potenciales a la investigación, en la cual se precisó bien la dirección de su domicilio y las posibilidades que tenían de que les hicieran un seguimiento adecuado.

A las mujeres que fueron elegidas para la investigación se les hizo una historia clínica completa y un examen ginecológico y los hallazgos se consignaron en un formulario especial. A continuación se suministró a cada una un paquete de linestrenol, $0.5 \mathrm{mg}$. por tableta, suficiente para 2 meses, con la instrucción de tomar una tableta diaria, y una tarjeta para anotar los días en que omitía la droga $y$ aquellos en que se produjera sangrado menstrual. Las mujeres fueron controladas cada mes, hasta completar un año de tratamiento.

Se investigaron los siguientes hechos:

a) Cambios en el ciclo menstrual.

b) Ocurrencia de los siguientes efectos colaterales: náuseas, cefálea, aumento o pérdida de peso, nerviosi- dad, sensibilidad mamaria, aborto, enfermedad tromboembólica, aumento o disminución de la líbido y otros efectos que ocurrieran con una frecuencia significativa como para merecer anotación.

c) La ocurrencia de retiros y sus causas.

d) Tasa de embarazos.

\section{Resultados}

308 mujeres ingirieron la droga por un total de 2.243 ciclos, discriminados en la forma que muestra el cuadro 1.

\section{CUADRO 1}

INGESTION CONTINUA DE 0.5 MG. DIARIOS DE LINESTRENOL, POR GRUPOS DE CICLOS COMPLETOS Y TOTAL DE CICLOS

\begin{tabular}{lc}
\hline $\begin{array}{c}\text { Grupos de ciclos } \\
\text { completos }\end{array}$ & $\begin{array}{c}\text { Total de ciclos } \\
\text { por grupo }\end{array}$ \\
\hline 5 o menos & 1.091 \\
Entre 6 y 10 & 732 \\
De 11 a 15 & 369 \\
De 16 a 19 & 51 \\
\hline
\end{tabular}

No ocurrió un solo embarazo debido a falla del método y sólo se presentó uno debido a falla de la mujer.

Como se aprecia en el cuadro anterior, casi la tercera parte de los ciclos ocurrió en el grupo de mujeres que usaron la droga por un total de 6 a 10, lo cual puede considerarse un tiempo relativametne corto para que se estabilice la respuesta del tracto genital. Con 5 ciclos o menos hay casi la mitad del total de ciclos estudiados, lo cual puede tener influencia en los tipos de respuesta observados. 
El cuadro 2 muestra cómo las $2 / 3$ partes de las mujeres eran menores de 30 años: 217 de 308 , es decir, se trataba de un grupo de mujeres de fertilidad alta.

\section{CUADRO 2}

DISTRIBUCION POR EDADES DE 308 MUJERES QUE TOMARON LINESTRENOL EN FORMA CONTINUA POR UN TOTAL DE 2.243 CICLOS

\begin{tabular}{|c|c|c|c|c|}
\hline Grupos & de & edades & Número & $\%$ \\
\hline Menos & de & 19 & 34 & 11.03 \\
\hline De 20 & $a$ & 24 & 107 & 34.74 \\
\hline De 25 & $\mathrm{a}$ & 29 & 76 & 24.67 \\
\hline $\mathrm{De} 30$ & $a$ & 34 & 56 & 18.18 \\
\hline De 35 & $a$ & 39 & 28 & 9.09 \\
\hline \multirow[t]{2}{*}{ De 40} & y & más & 7 & 2.27 \\
\hline & & & 308 & 100.00 \\
\hline
\end{tabular}

El siguiente cuadro muestra que 254 de 308 mujeres, un $82.46 \%$ tenían ciclos entre 28 y 31 días de duración (Cuadro 3 ).

\section{CUADRO 4}

CAMBIOS EN LA CANTIDAD DEL FLUJO MENSTRUAL EN 308 PACIENTES QUE TOMARON LINESTRENOL EN FORMA CONTINUA POR UN TOTAL DE 2.243 CICLOS

\begin{tabular}{lcc}
\hline Tipo de cambio & Número & $\%$ \\
\hline Disminución & 423 & 18.85 \\
Aumento & 453 & 20.19 \\
No cambió & 1.367 & 60.94 \\
& $\overline{2.243}$ & 100.00 \\
\hline
\end{tabular}

\section{CUADRO 3}

DISTRIBUCION DE LA DURACION DE LOS CICLOS MENSTRUALES EN 308 MUJERES, PREVIAMENTE A LA INICIACION DE LA INGESTION DE LINESTRENOL EN FORMA CONTINUA

\begin{tabular}{lrr}
\hline Duración & Número & $\%$ \\
\hline Menos de 20 días & 2 & 0.64 \\
$20-21$ & 4 & 1.29 \\
$22-23$ & 1 & 0.32 \\
$24-25$ & 9 & 2.92 \\
$26-27$ & 14 & 4.54 \\
$28-29$ & 82 & 26.62 \\
$30-31$ & 172 & 55.84 \\
$32-33$ & 4 & 1.29 \\
$34-35$ & 8 & 2.59 \\
Más de 35 & 12 & 3.89 \\
& 308 & 100.00 \\
\hline
\end{tabular}

Con la ingestión de la droga, hubo una tendencia ligera a un aumento en la duración de la cantidad del flujo menstrual como lo demuestra el cuadro 4.

En $11 \%$ de los casos los ciclos no experimentaron variaciones en la duración y en $25 \%$ tal variación fue de 2 días más o menos, lo cual en realidad se considera una fluctuación dentro de los límites normal. La ocurrencia de hemorragia irregular o sangrado anormal fue descendiendo desde los primeros 3 ciclos, en que ocurrió entre un 40 y un $26.6 \%$ para ser de un $17.6 \%$ en el ciclo $6 \%$, un $5 \%$ en el $15 \%$ y aún desaparecer en el último. Cuadro 5.

\section{CUADRO 5}

OCURRENCIA DE HEMORRAGIA IRREGULAR DE ACUERDO AL ORDEN DE LOS CICLOS EN 308 PACIENTES QUE TOMARON LINESTRENOL EN FORMA CONTINUA POR UN TOTAL DE 2.243 CICLOS

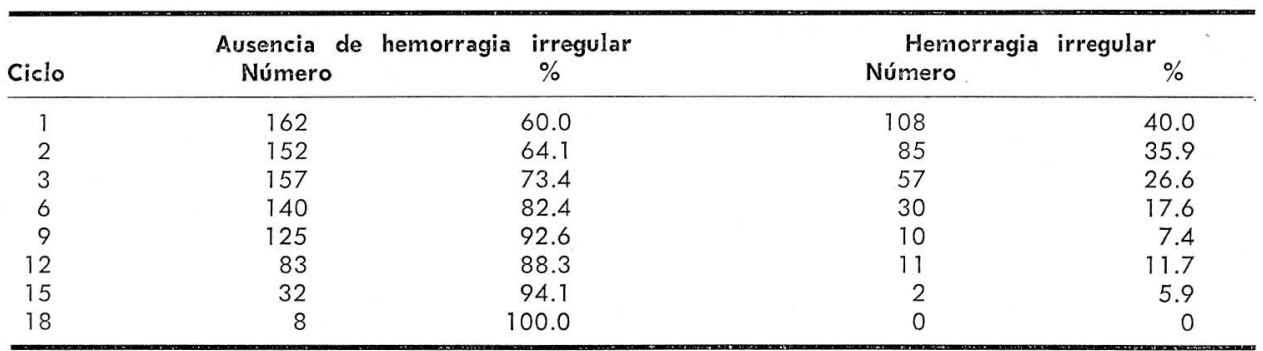


Los efectos colaterales fueron moderados y muchos de ellos de un carácter subjetivo, como por ejemplo la nerviosidad, y es difícil responsabili- zar a la droga directamente por su ocurrencia; son también síntomas difíciles de puntualizar en una paciente dada. Cuadro 6.

\section{CUADRO 6}

EFECTOS COLATERALES DE LA INGESTION DE 0.5 MG. DE LINESTRENOL EN FORMA CONTINUA POR UN TOTAL DE 2.243 CICLOS, EN 308 MUJERES

\begin{tabular}{|c|c|c|c|}
\hline Tipo de efecto & $\begin{array}{l}\mathrm{N}^{0} \text { de mujeres que } \\
\text { se quejaron al menos } \\
\text { una vez }\end{array}$ & $\begin{array}{l}N^{0} \text { de ciclos } \\
\text { con una queja }\end{array}$ & $\begin{array}{l}\% \text { del número } \\
\text { total de ciclos }\end{array}$ \\
\hline Náusea & 34 & 50 & 2.2 \\
\hline Cefálea & 119 & 257 & 11.5 \\
\hline Aumento de peso de 1 kilo & 88 & 155 & 6.9 \\
\hline Disminución de peso: 1 kilo & 105 & 225 & 10.0 \\
\hline Nerviosidad & 129 & 305 & 13.6 \\
\hline Sensibilidad mamaria & 27 & 33 & 1.5 \\
\hline Aborto & 0 & 0 & 0 \\
\hline Enfermedad trombo embólica & 0 & 0 & 0 \\
\hline Aumento de la líbido & 3 & 3 & 0.1 \\
\hline Disminución de la líbido & 14 & 17 & 0.8 \\
\hline
\end{tabular}

En 38 casos hubo abandono del método debido a que los efectos colaterales fueron considerados excesivos por la usuaria.

Las causas de retiro se discriminaráin en el Cuadro 7.

\section{Conclusiones}

1. La administración del linestrenol en forma continua a la dosis de $0.5 \mathrm{mg}$. tiene tendencia a producir pocos cambios en los ciclos menstruales; los más salientes son una tendencia a aumentar en días el flujo menstrual y algunas irregularidades en los ciclos. Estas últimas disminuyen gradualmente a medida que aumenta el número de ciclos administrados.

2. No ocurrieron efectos colaterales graves y los que ocurrieron sólo obligaron a $12.3 \%$ de las mujeres a abandonar el método.

3. No ocurrieron embarazos en este grupo de mujeres.

\section{Resumen}

Se presentan los resultados de la administración de linestrenol $0.5 \mathrm{mg}$. en forma continua a 308 mujeres, hasta completar un total de $2.243 \mathrm{ci}$ clos y se analizan sus efectos sobre la menstruación, la ocurrencia de efectos colaterales y su eficacia anticonceptiva.

\section{Summary}

Lynestrenol $0.5 \mathrm{mg}$. administered in a continuous form determines few changes in the menstrual cycles of the women who take it. The most important are a tendency to increase the days of menstrual flow and some cycles irregularities.

The last one decrease gradually as the number of cycles increase.

No serious side affects were observed and those that ocurred influenced only 38 women to change the method.

No pregnancies ocurred in this group of women. 


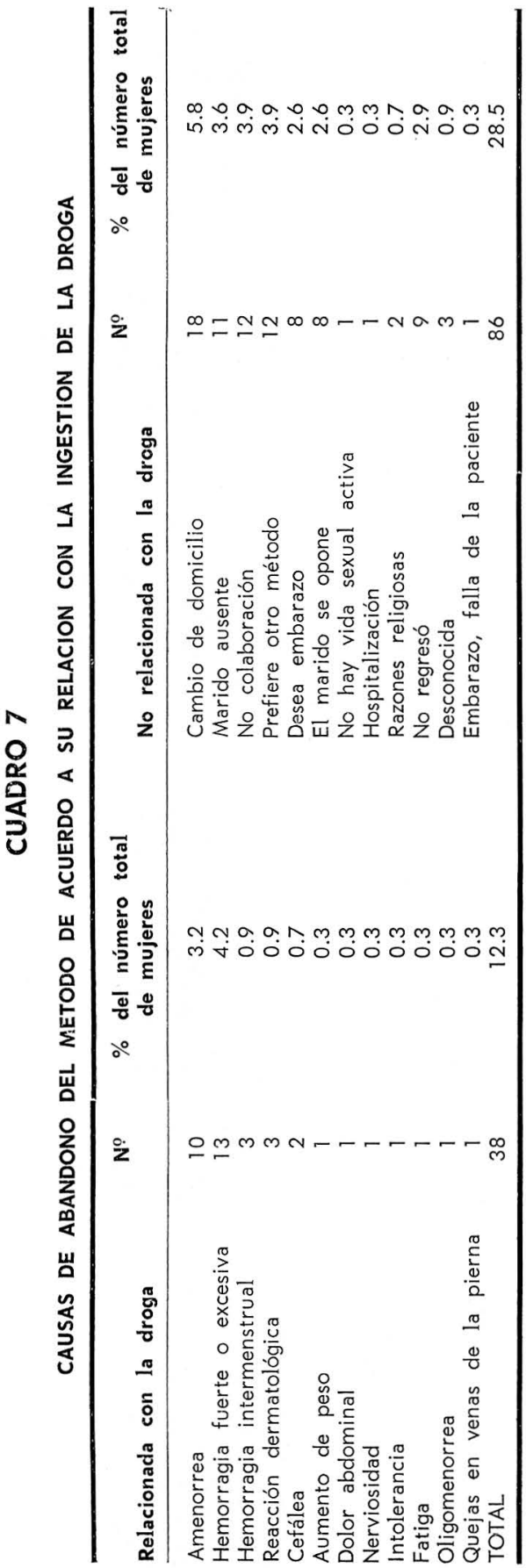

\section{AGRADECIMIENTOS}

Al personal de médicos de la Clínica de Maternidad de Medellín, Luz Castro de Gutiérrez y del Departamento de Obstetricia y Ginecología del Hospital Universitario San Vicente de Paúl, por su colaboración en la evaluación clínica de las mujeres que participaron en este estudio.

\section{BIBLIOGRAFIA}

1 MARTINEZ MANAUTOU, J., CORTES, V. \& GINER, J.: Low doses of progestogen as an * approach to fertility control. Fertil. \& Steril. 17 : 49-57, 1966.

2 BOTERO, J., SANCHEZ, F., HENAO, G. \& DUQUE, LUIS F.: Acetato de clormadinona en microdosis como anticonceptivo. Rev. Col. Obstet. Ginec. 21 : 503-508, 1970.

3 JACKSON, M. C. N.: Experience wtih sequential and low dose progestogen oral contraceptives. Lancet. 2 (7619): 540-541, 1969.

4 SALAZAR, R., CACERES, E., \& LOPEZ, G. : Ensayo del acetato de lynestrenol a dosis baja y continua como anticonceptivo. Trabajo presentado al $X$ Congreso Colombiano de Obstetricia y Ginecología. Cali, Diciembre 48, 1973.

5 RAVN, J. Contraception with the Lynestrenol "Mini-Pill". Arzneim Forsch, (Drug Res) 22 : 104-113, 1972.

6 KREMER, J., BENNEN, J. \& HISJES, J. H. De Mini-Pill. Med. Tijdschr. Geneesk. 113: 2324-2329, 1969.

7 VANDEKERCKHOVE, D., DEMOL, R. \& LAPOUTRE, L. Continuous low dosage progestagen treatment. Preliminary results with the use of Lynestrenol $0.5 \mathrm{mg}$. En: "Investigations in Sterility". Proc. of the First European Congress on Steriliyt. Venice, Mayo 6-7, 1967, pág. 51-58.

8 SCHIDT-ELMENDROFF, H., KAISER, E. \& KOPERA, $H$. The effect of $0.5 \mathrm{mg}$. Lynestrenol on the follicle estimulating hormone, luteinizing hormone oestrogen and pregnanediol excretion during the menstrual cycle. Arzneimittel Forsch. 19: 1277-1279, 1969. 